\title{
The Role of the Globular Heads of C1q Receptor (gC1qR) Gene in Regulating Apoptosis of Human Cervical Squamous Cell Carcinoma
}

\author{
Zhu Liu, Ya-juan Sub,d Ping-qing Guc Zhong-yi Jia Xi-guo Wang ${ }^{\mathrm{a}}$ \\ Ling-juan $\mathrm{Gao}^{\mathrm{c}}$ \\ ${ }^{a}$ Clinical Laboratory, Huangdao District of Traditional Chinese Medicine, Qingdao; ${ }^{b} \mathrm{Clinical}$ \\ Laboratory, the Third Affiliated Hospital of Harbin Medical University, Harbin; ' State Key Laboratory \\ of Reproductive Medicine, Department of Clinical Laboratory, Nanjing Maternity and Child Health \\ Care Hospital Affiliated to Nanjing Medical University, Nanjing; ${ }^{\text {d }}$ Zhu Liu and Ya-juan Su \\ contributed equally to this work
}

\section{Key Words}

Globular heads of $\mathrm{C} 1 \mathrm{q}$ receptor $(\mathrm{gClqR}) \cdot$ Apoptosis $•$ p38 MAPK

\begin{abstract}
Background: The globular heads of the human $\mathrm{C} 1 \mathrm{q}$ receptor $(\mathrm{gCl} \mathrm{qR})$ are multi-compartmental and multi-functional cellular proteins. The list of biological responses mediated by the $\mathrm{gClqR}$ includes growth perturbation and morphological abnormalities, along with the initiation of apoptosis. However, the effects of the gC1qR on the apoptosis of cervical squamous carcinoma cells ( $\mathrm{C} 33 \mathrm{a}$ and $\mathrm{SiHa}$ ) have not been demonstrated. Methods: Here, human cervical tissues were examined for the expression of the gC1qR using real-time PCR and Western blot analysis. Apoptotic death of C33a and $\mathrm{SiHa}$ cells was assessed by flow cytometric analysis to detect the subG1 population. Viability, migration and proliferation of C33a and SiHa cells were detected via the water-soluble tetrazolium salt (WST-1) assay, the Transwell assay and the ${ }^{3} \mathrm{H}$-thymidine incorporation into DNA assay $\left({ }^{3} \mathrm{H}-\mathrm{TdR}\right)$, respectively. Results: These data showed that expression of the $\mathrm{gClqR}$ protein was significantly decreased in human cervical squamous cell carcinoma tissues relative to normal cervix tissues. C33a and SiHa cells transfected with a GFP-gClqR vector resulted in the up-regulation of cellular apoptosis and an apparent increase in the expression of the p38 mitogen-activated protein kinase (p38 MAPK). Further, the changes in C33a and SiHa cells viability, migration and proliferation observed upon overexpression of gC1qR could be abrogated using the p38 MAPK inhibitor SB202190. Conclusion: These data indicate that $\mathrm{gClqR}$ inhibits viability, migration and proliferation of cervical squamous cells carcinoma via the p38 MAPK signalling pathway.
\end{abstract}


Liu/Su/Gu/Ji/Wang/Gao: gC1qR-regulated Apoptosis of Cancer Cells

\section{Introduction}

Cervical cancer is the second most common malignant neoplasm in females and is the leading cause of death from cancer in many low-resource countries [1, 2]. The lack of preventive strategies, early diagnostic methods, and effective therapies to treat recurrent cervical tumours creates a pressing need to understand the pathogenesis of cervical cancer and to identify molecular markers and targets for its diagnosis and therapy [3]. The unlimited growth and metastasis of cervical tumours are the main causes of cancer-related deaths resulting from the failure of treatment of cervical cancer [4]. However, this unlimited growth and metastasis involves the expression of both pro- and anti-apoptotic proteins that remain largely unknown.

Apoptosis is an actively regulated process of cell death necessary for proper control of tissue growth. Apoptosis is believed to play a role in many physiological processes and, when defective, can contribute to the pathogenesis of cancer $[5,6]$. The receptor for the globular heads of $\mathrm{C} 1 \mathrm{q}(\mathrm{gC} 1 \mathrm{qR})$ is a ubiquitous and highly anionic cellular protein of $33 \mathrm{kDa}$ that was initially identified as a protein of the mitochondrial matrix [7]. Representing a novel mechanism, evidence that $\mathrm{gC} 1 \mathrm{qR}$ induces T-cell dysfunction includes the induction of the suppressor of cytokine signalling (SOCS), a powerful inhibitor of cytokine signalling [8]. Indeed, the gC1qR mediates many biological responses, including inflammation, infection and immune regulation [9]. Examples of such responses include growth perturbation and morphological abnormalities, along with the initiation of apoptosis [10]. Therefore, our present study aimed to investigate the effects of $\mathrm{gC} 1 \mathrm{qR}$ protein expression on cervical cancer cell apoptosis and the related signalling pathway.

\section{Materials and Methods}

\section{Reagents}

The C33a and SiHa cervical cancer cell lines were obtained from Boster Technology (Wuhan, HuBei, China). Human cervical epithelial cell line CRL2614 was purchased from American Type Culture Collection (ATCC). Lipofectamine 2000 was purchased from Invitrogen (Carlsbad, CA, USA). The Phototope-HRP Western Blot Detection System, including an anti-mouse IgG, an HRP-linked antibody, a biotinylated protein ladder, 20X LumiGLO Reagent and 20X peroxide, were purchased from Cell Signaling Technology (Beverly, MA, USA). The SB202190 p38 MAPK inhibitor was obtained from Sigma-Aldrich Inc (St. Louis, MO, USA). The Annexin V-FITC/ Propidium Iodide Flow Cytometry Assay Kit were purchased from Invitrogen (Carlsbad, CA, USA). Antibodies targeting gC1qR, p38 MAPK, phospho-p38 MAPK and actin were products of Santa Cruz (Santa Cruz, CA, USA) and Cell Signaling Technology. Cell culture supplies were purchased from Life Technologies (Gaithersburg, MD, USA). Unless otherwise specified, all other reagents were of analytical grade.

\section{Tissue Procurement and Preparation}

Human cervical cancer tissues were collected from 30 patients who underwent radical hysterectomy because of cervical carcinoma at Nanjing Maternity and Child Health Care Hospital between October 2007 and January 2010. Tumour specimens were obtained immediately after surgery. Local ethical approval was obtained before commencing this study and, as appropriate, tissue was collected with informed consent. The human cervical squamous cells carcinoma tissues and normal cervix from patients who have had a hysterectomy for non cancer diagnoses were reviewed by a pathologist and histologically confirmed.

\section{Cell Culture and DNA Transfection Conditions}

Cells were cultured in Dulbecco's modified Eagle's medium/Ham's F-12 medium containing 10\% foetal bovine serum and $5 \mu \mathrm{g} / \mathrm{mL}$ insulin in a $37{ }^{\circ} \mathrm{C}$ incubator with $5 \% \mathrm{CO}_{2}$. Complementary DNA (cDNA) to $\mathrm{gC1}$ qR was constructed in frame using the BamHI/EcoRI sites of the pEGFP-C1 vector. The resulting GFP$\mathrm{gC1} \mathrm{qR}$ vector was then transfected into $\mathrm{C} 33 \mathrm{a}$ and SiHa cells. According to the vendor's protocol, $500 \mathrm{pmol}$ of GFP-gC1qR vector and Lipofectamine $2000(10 \mu \mathrm{L})$ were diluted in $750 \mu \mathrm{L}$ of OptiMEM (Life Technologies, 
Liu/Su/Gu/Ji/Wang/Gao: gC1qR-regulated Apoptosis of Cancer Cells

Gaithersburg, MD, USA). After preincubation for $45 \mathrm{~min}$ at $37^{\circ} \mathrm{C}$, the solutions were mixed and incubated for an additional $15 \mathrm{~min}$ at room temperature. The Lipofectamine 2000/GFP-gC1qR vector mixture was subsequently overlaid onto the cells and incubated for $2 \mathrm{~h}$. Finally, $1 \mathrm{~mL}$ of growth medium (20\% FCS) per well was added for further cultivation of the cells. Reporter gene activities were normalised to total protein, and all results represent the average of triplicate experiments.

\section{Real-time Quantitative Polymerase Chain Reaction}

Total RNA was isolated from tissue using Trizol reagent (Invitrogen, Carlsbad, CA, USA) according to the manufacturer's instructions. The isolated RNA was then DNase-treated and reverse-transcribed according to the manufacturers instructions. For detection of gC1qR expression, Primer-F (5'-AAT CAC ACG GTA GAC ACT GAA ATG CC-3') and Primer-R (5'-CAT CAT CCC ATC TAA AAT GTC CCC TG-3') were used with the FAM/TAMRA-labelled probe $5^{\prime}$-TGC TCC AGT TCA ACC AAC GTC CTT CTC-3'. $\beta$-actin was quantified using Primer-F (5'-TCA CCC ACA CTG TGC CCA TCT ATG A-3') and Primer-R (5'-CAT CGG AAC CGC TCA TTG CCG ATA G-3') with the FAM/TAMRA-labelled probe 5'-ACG CGC TCC CCC ATG CCA TCC TGC GT-3'. Real-time PCR was performed using an ABI PRISM 7300 sequence detection system with the following thermal cycling conditions: $2 \mathrm{~min}$ at $50^{\circ} \mathrm{C}$ and $10 \mathrm{~min}$ at $95^{\circ} \mathrm{C}$, followed by a total of 40 cycles of $15 \mathrm{~s}$ at $95^{\circ} \mathrm{C}$ and $1 \mathrm{~min}$ at $60^{\circ} \mathrm{C}$. All reactions were performed in $50 \mu \mathrm{L}$ reaction volumes in triplicate. Standard curves were generated for $\mathrm{gC} 1 \mathrm{qR}$ and $\beta$-actin. The $\beta$-actin gene was used as an internal control in all PCR experiments. Relative amounts of gC1qR mRNA were normalised to $\beta$-actin mRNA using the following formula: $2^{-\Delta \Delta C T}=2^{- \text {(CT.g.g1qR- }}$ CT.actin)Time $\mathrm{x}+($ CT.gC1qR- CT.actin) Time 0 .

\section{Western Blot Analysis}

Following specific treatments, the C33a and SiHa cells were collected and placed in sample buffer and then incubated in lysis buffer containing $150 \mathrm{mM} \mathrm{NaCl}, 1 \mathrm{mM} \mathrm{Na}_{3} \mathrm{VO}_{4}, 50 \mathrm{mM} \mathrm{NaF}, 1 \%$ Triton X-100, 1 mM EDTA, $1 \mathrm{mM}$ PMSF, $10 \%$ glycerol, $20 \mathrm{mM}$ Tris- $\mathrm{HCl}$ (pH 7.5) and protease inhibitors for $30 \mathrm{~min}$ on ice. The supernatants were collected following centrifugation at $13,000 \times \mathrm{g}$ at $4{ }^{\circ} \mathrm{C}$ for $15 \mathrm{~min}$. Equal amounts of protein were separated by SDS-PAGE on a $10-15 \%$ polyacrylamide gel and transferred onto a PVDF membrane. The membranes were then blocked for $1 \mathrm{~h}$ in 5\% non-fat milk in PBST (PBS containing 0.05\% Tween 20) and incubated with the appropriate primary antibodies and horseradish peroxidase-conjugated secondary antibodies. The protein bands were visualised using the enhanced chemiluminescence (ECL) Western Detection System.

\section{Cell Viability Assay}

We confirmed proliferative activity using the water-soluble tetrazolium salt (WST-1) assay (Roche Diagnostics, Mannheim, Germany). The WST-1 assay is a colorimetric method in which the dye intensity is proportional to the number of viable cells. Cells were seeded into 96-well microtiter plates at a concentration of $5 \times 10^{3}$ cells/well. After $12 \mathrm{~h}$, cells were treated with different media for $48 \mathrm{~h}$, washed with PBS, and the cell proliferation reagent WST-1 was added and incubated for $4 \mathrm{~h}$. Sample absorbance was analysed with a bichromatic ELISA reader at $450 \mathrm{~nm}$. All experiments were performed in triplicate with different passages of the C33a and SiHa cells.

\section{In Vitro Migration Assay}

Cell migration was assayed using 24-mm diameter chambers with 8- $\mu \mathrm{m}$ pore filters (Transwell, 6-well cell culture). The C33a and SiHa cells were removed from the culture flasks, resuspended at $7.5 \times 10^{6}$ cells/ $\mathrm{mL}$ in serum-free medium, and $0.2 \mathrm{~mL}$ of the cell suspension was added to each upper chamber. Different medium $(0.5 \mathrm{~mL})$ was then added to the lower chambers. The chambers were incubated for $48 \mathrm{~h}$ at $37^{\circ} \mathrm{C}$ in a humidified atmosphere containing $5 \% \mathrm{CO}_{2}$. The filters were then fixed in $95 \%$ ethanol and stained with hematoxylin-eosin (H.E.). The upper surfaces of the filters were scraped twice with cotton swabs to remove non-migrated cells. Experiments were repeated in triplicate with different passages of the C33a and SiHa cells, and migrated cells were counted microscopically (400 $\times$ magnification) in five different fields per filter.

Measurement of ${ }^{3} \mathrm{H}$-thymidine Incorporation ( $\mathrm{\beta} H-\mathrm{TdR}$ )

Cells were plated into 96-well plates and incubated overnight. Medium was removed from the cells and replaced with $200 \mu \mathrm{L}$ of the appropriate medium, as described in the results section. The cells were 


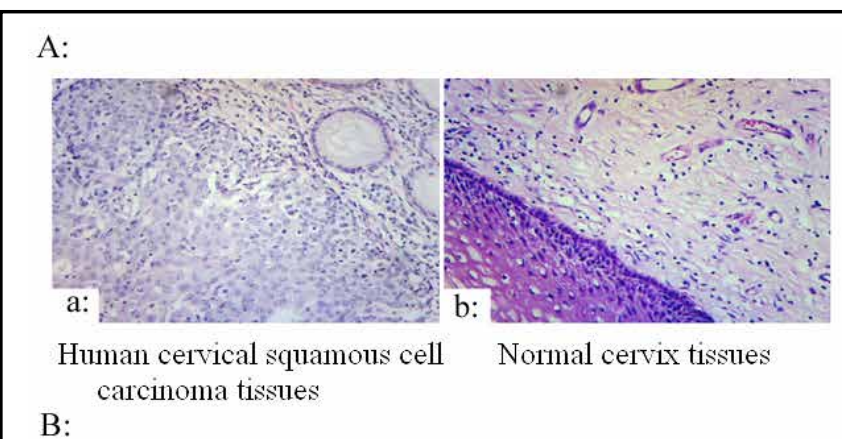

C:

a:

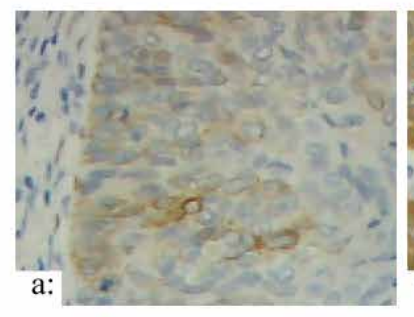

Human cervical squamous cell carcinoma tissues

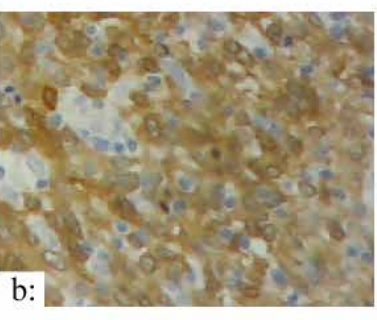

Normal cervix tissues

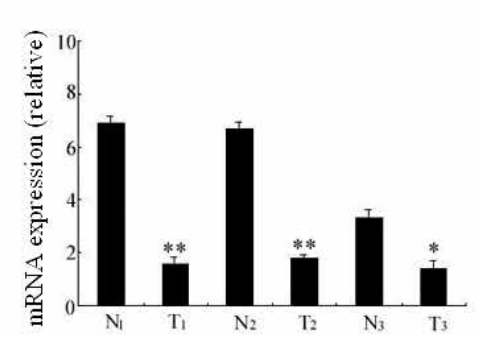

b:

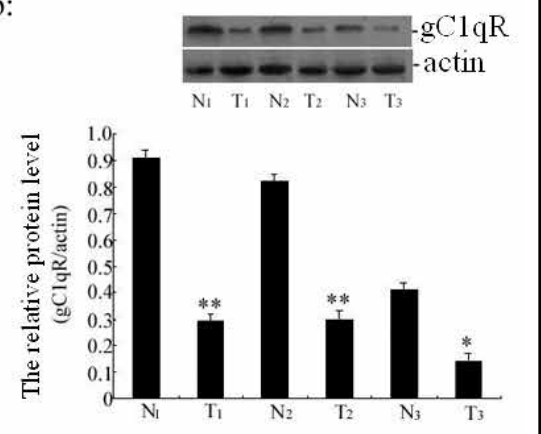

Fig. 1. The gC1qR levels in human cervical tissue. A: The human cervical tissues pathologic characterization. The human cervical squamous cells carcinoma tissues and normal cervix from patients who have had a hysterectomy for non cancer diagnoses were observed by electronic microscope. a: Cervical squamous cells carcinoma were seen in cervical carcinoma tissues $(\times 200)$; b: No ultrastructural evidence of cervical squamous cells carcinoma in normal cervix tissues $(\times 200)$. B: The results of immunohistochemical staining. The positive results for $\mathrm{gC} 1 \mathrm{qR}$ antigen in human cervical tissues by immunohistochemistry $(\times 200)$. a: staining of monoclonal anti-gC1qR antibody in human cervical squamous cells carcinoma tissues; b: staining of monoclonal anti-gC1qR antibody in normal cervix tissues. C: Relative gC1qR expression levels are shown between human cervical squamous cells carcinoma tissues (T) and normal cervix from patients who have had a hysterectomy for non cancer diagnoses $(\mathrm{N})$. a: The mRNA expression level of gC1qR were analysed by real-time PCR. b: The expression of the gC1qR protein was measured using Western blot analysis. The graph displays the relative $\mathrm{gC} 1 \mathrm{qR}$ protein levels normalised to $\beta$-actin. The gC1qR gene in human cervical cancer tissue had low expression. The results shown are the mean \pm SD of three independent experiments $(n=3)$, each conducted in triplicate. Student's t-test: $\mathrm{N}$ versus T. ${ }^{* *} p<0.01,{ }^{*} p<0.05$.

incubated for $54 \mathrm{~h}$, and then DNA synthesis was determined by ${ }^{3} \mathrm{H}-\mathrm{TdR}$ for the final $18 \mathrm{~h}$. The media were carefully removed, and the cells were detached with $50 \mu \mathrm{L}$ of trypsin-EDTA. The cells were then harvested onto glass filters with a Tomtech cell harvester, and the radioactivity retained on the dried filters was measured by the addition of $50 \mathrm{~mL}$ of scintillation liquid and counting in a TopCount NxT scintillation counter. All experiments were performed in triplicate with different passages of the C33a and SiHa cells.

\section{Detection of Apoptotic Cells}

Annexin V-FITC/propidium iodide staining was utilised to detect apoptotic C33a and SiHa cells by flow cytometric analysis. After different treatments at the indicated times, cells were washed and resuspended with binding buffer ( $2.5 \mathrm{mM} \mathrm{CaCl}_{2}, 10 \mathrm{mM}$ HEPES, pH 7.4, and $140 \mathrm{mM} \mathrm{NaCl}$ ) before being transferred to a $5 \mathrm{~mL}$ tube. The cells were incubated in the dark with $5 \mu \mathrm{L}$ each of annexin and propidium iodide for $15 \mathrm{~min}$. Binding buffer was then added to each tube, and the samples were analysed using a Beckman Coulter Epics XL flow cytometer.

\section{Statistical Analysis}

Most results are presented as means \pm SD. Differences between various data sets were tested for significance using Student's t-test, and a $p$-value of less than 0.05 was considered significant $\left({ }^{* *} p<0.01 ;{ }^{*} p\right.$ $<0.05 ;{ }^{\#} p>0.05$ ). 
Fig. 2. The levels of gC1qR expression. A: Relative gC1qR gene expression levels are shown in cervical squamous carcinoma cell line, C33a, SiHa and human cervical epithelial cell line, CRL2614. The different expression level of gC1qR were analysed by real-time PCR as described. B: The expression of the $\mathrm{gC} 1 \mathrm{qR}$ protein was measured by Western blot analysis. The graph depicts the relative gC1qR protein levels normalised to actin. The results are expressed as the mean \pm SD of three separate experiments. ${ }^{* *} p<0.01$ versus CRL2614 cells; ${ }^{*} p<0.05$ versus CRL2614 cells.
A:

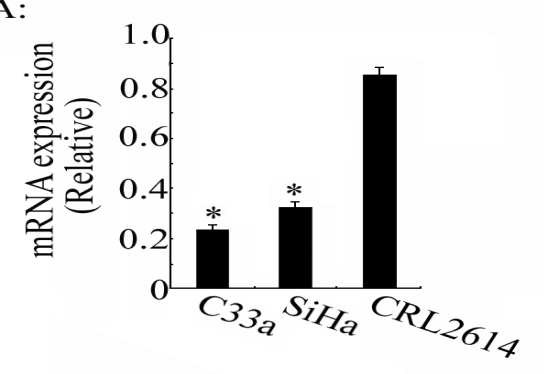

B:
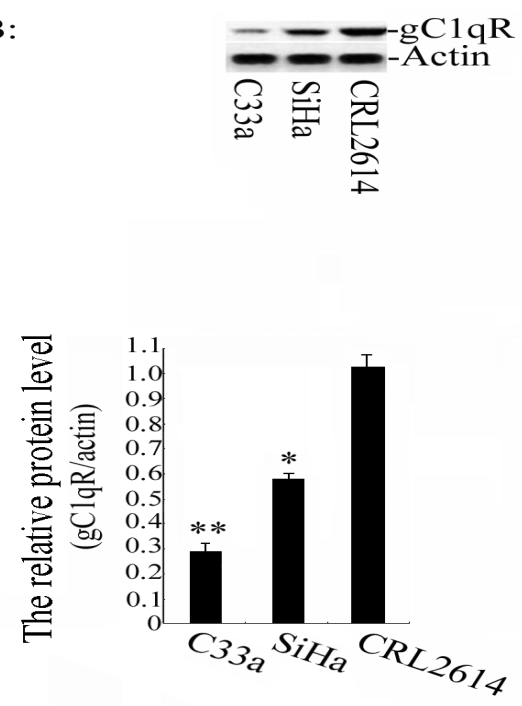

\section{Results}

The expression of the gC1qR gene in human cervical tissue

To investigate the relationship between the expression of the $\mathrm{gC1qR}$ gene and human cervical squamous cells carcinoma, the gC1qR expression levels of 30 cases of human cervical squamous cells carcinoma and 30 cases of normal cervix from patients who have had a hysterectomy for non cancer diagnoses were analysed in this experiment. The pathologic characterization of human cervical tissues was confirmed squamous cells carcinoma by electronic microscope (Fig. 1A-a). As shown in Figure 1B, the gC1qR expression was analyzed by immunohistochemistry in human cervical tissues. The gC1qR expression was significantly decreased in human cervical squamous cell carcinoma tissues, comparing with normal cervix tissues. Meanwhile, the gC1qR expression was further analyzed by real-time PCR and Western blot analysis (Fig. 1C). The results showed that the expression levels of gC1qR mRNA and protein were significantly decreased in human cervical squamous cells carcinoma tissues $(\mathrm{T})$ compared with normal cervix tissues $(\mathrm{N})$. This finding suggested that gC1qR might play a negative role in the survival of human cervical squamous cells carcinoma.

\section{Identification and quantification of gC1qR expression}

In this experiment, we determined the $\mathrm{gC} 1 \mathrm{qR}$ expression levels in cervical squamous carcinoma cell line, C33a, SiHa and human cervical epithelial cell line, CRL2614. Real-time PCR and Western blot results showed that the level of gC1qR expression in C33a and SiHa cells was lower when compared with CRL2614 cervical epithelial cell line. The C33a and SiHa cells were the primary subject in subsequent experiments. 


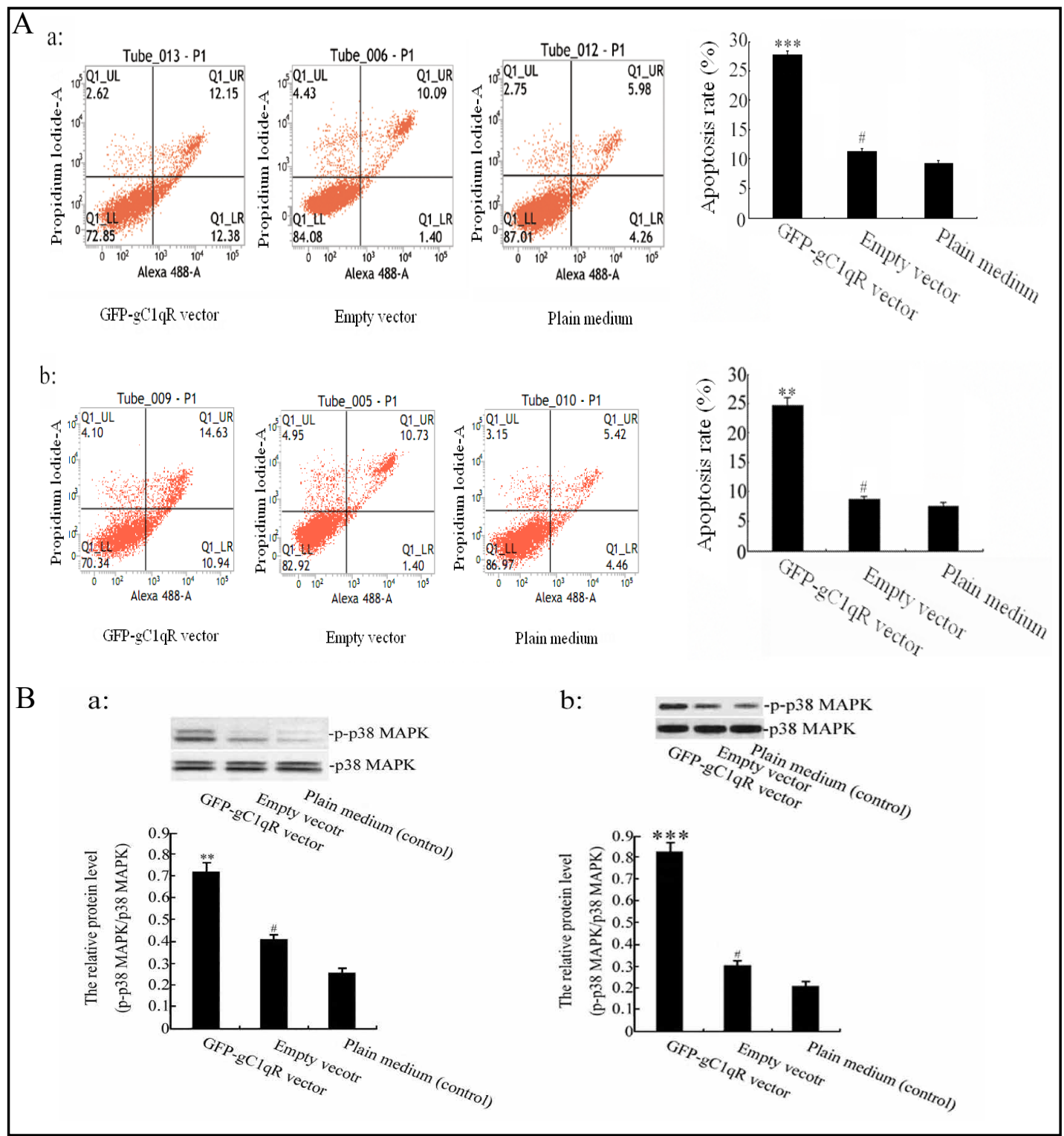

Fig. 3. Effects of gC1qR overexpression on C33a and SiHa cells. A: The apoptotic death of cells. C33a cells (a) and SiHa cells (b) were transfected with the GFP-gC1qR vector, empty vector, or plain medium. At 48 $\mathrm{h}$ post-transfection, the apoptotic death of cells was assessed by flow cytometric analysis to detect the subG1 population. ${ }^{* * *} p<0.001$ versus the plain medium group; ${ }^{* *} p<0.01$ versus the plain medium group; $\# p>0.05$ versus plain medium group (control). B: Effects of gC1qR overexpression on activation of p38 MAPK signal pathway in C33a and SiHa cells. Western blot analyses of phospho-p38 MAPK protein in C33a cells (a) and SiHa cells (b) treated with GFP-gC1qR vector, empty vector, or plain medium. At 48 h posttransfection, the p38 MAPK protein was detected by Western blot analysis as described in "Meterial and Methods" The relative protein level of phospho-p38 MAPK was normalised to total p38 MAPK. ${ }^{* * *} p<0.001$ versus the plain medium group; ${ }^{* *} p<0.01$ versus the plain medium group; ${ }^{*} p>0.05$ versus plain medium group (control).

Functional analysis of $g C 1 q R$ treatment of the C33a and SiHa cells

To determine if the accumulation of gC1qR could trigger apoptotic death, the apoptotic death of C33a and SiHa cells was assessed by flow cytometry following treatment with the GFP-gC1qR vector or empty vector for $48 \mathrm{~h}$. Figure $3 \mathrm{~A}$ shows that accumulated gC1qR could 
Fig. 4. Human cervical cancer cell viability, migration and proliferation. Viability, migration and proliferation of C33a and $\mathrm{SiHa}$ cells were detected in this experiment. At $48 \mathrm{~h}$ post-transfection with the GFP-gC1qR vector, cells $(3 \times$ $10^{4} / \mathrm{mL}$ ) were treated with $10 \mu \mathrm{M}$ SB202190 (a p38 MAPK inhibitor) for $18 \mathrm{~h}$. A: C33a cells (a) and SiHa cells (b) viability was detected via the WST-1 assay. Sample absorbance was analysed using a bichromatic ELISA reader at $450 \mathrm{~nm}$. "* $p<$ 0.01 versus plain medium group; ${ }^{*} p<0.05$ versus plain medium group; $" ~ p>0.05$ versus plain medium group. B: The migration of C33a cells (a) and SiHa cells (b) was measured by Transwell assay. Migrated cells were counted microscopically $(400 \times)$ in five different fields per filter. ${ }^{* *} p<0.01$ versus plain medium group; ${ }^{*} p<$ 0.05 versus plain medium group; ${ }^{*} p>0.05$ versus plain medium group. C: The
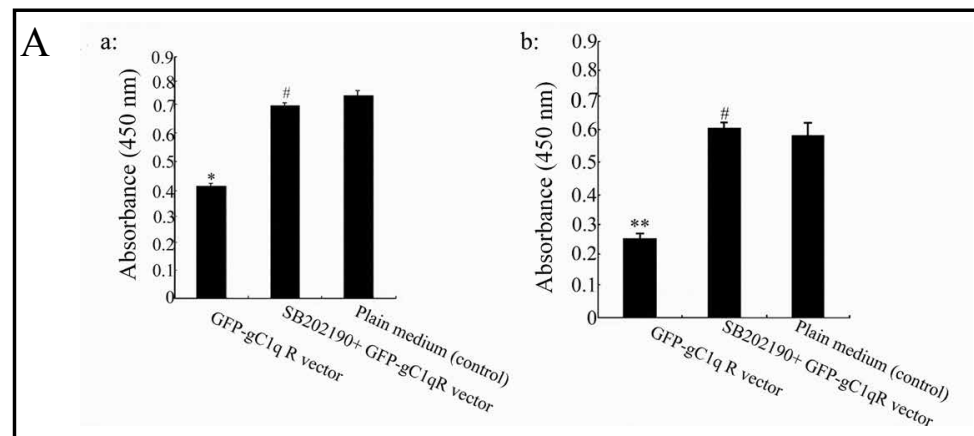

B
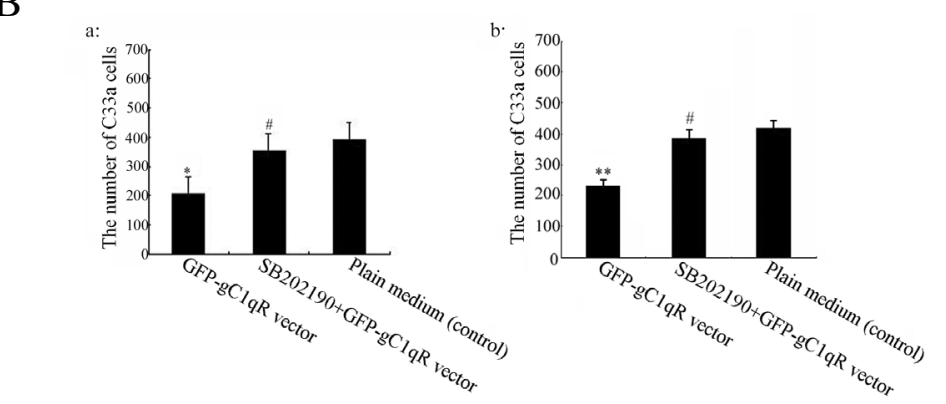

$\mathrm{C}$ a:
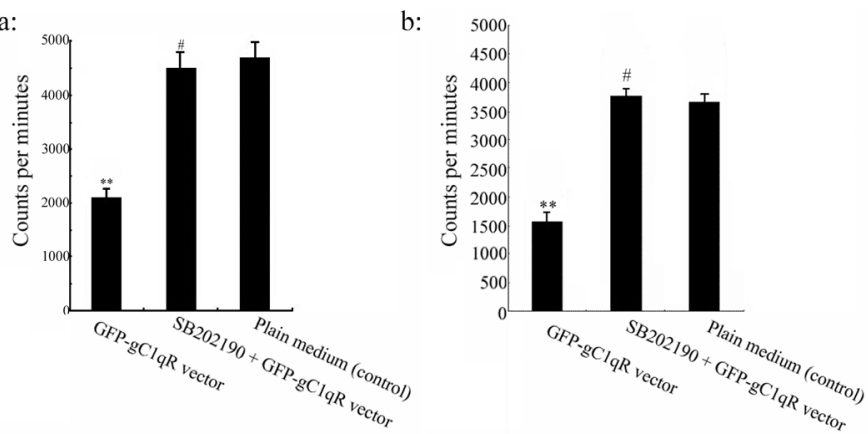

incorporation of ${ }^{3} \mathrm{H}$-thymidine into DNA was measured over the last $18 \mathrm{~h}$ of the final incubation in C33a cells (a) and SiHa cells (b). The results represent the mean \pm SD from 3 independent experiments. ${ }^{* *} p<0.01$ versus plain medium group; ${ }^{\#} p>0.05$ versus plain medium group.

increase the number of cells in the subG1 population in the GFP-gC1qR-transfected group. However, the subG1 population of in the empty vector-transfected cells showed only a slight increase when compared with the plain medium group. Additionally, we evaluated the activation of phospho-p38 MAPK. As shown in Figure 3B, phospho-p38 MAPK was observed after GFP-gC1qR vector, empty vector, and plain medium treatment in C33a and SiHa cells for $48 \mathrm{~h}$. The phospho-p38 MAPK protein was notably increased in cells transfected with the GFP-gC1qR vector when compared with the plain medium group. In contrast, a slight change in phospho-p38 MAPK expression was found in cells treated with empty vector and plain medium alone. These results suggested that gC1qR-induced apoptotic cell death was related to activation of the p38 MAPK signalling pathway.

The p38 MAPK inhibitor SB202190 reverses the gC1qR-induced viability, migration and proliferation of human cervical cancer cells

To investigate the involvement of the p38 MAPK signalling pathway in the gC1qR-induced viability, migration and proliferation of human cervical squamous carcinoma cells, C33a and SiHa cells were transfected with the GFP-gC1qR vector, SB202190 + the GFP-gC1qR vector, or plain medium. Cell viability was determined by the WST-1 assay. As shown in Figure 4A, 
transfection with the GFP-gC1qR vector decreased cell viability when compared with the plain medium group, but the viability of cells in the SB202190 + GFP-gC1qR vector group did not differ from that of the plain medium group. The p38 MAPK inhibitor therefore reversed the gC1qR-induced viability decrease of C33a and SiHa cells.

To determine if the p38 MAPK signalling pathway is involved in gC1qR-induced cell migration, C33a and SiHa cells were transfected with the GFP-gC1qR vector, SB202190 + GFP-gC1qR vector, or plain medium. The number of migrated cells in the group treated with the GFP-gC1qR vector was significantly lower than that in the plain medium group $(p<0.05)$. There were no differences in the numbers of migrated cells between the SB202190 + GFPgC1qR vector group and the plain medium group (Fig. 4B, $p>0.05$ ).

As shown in Figure 4C, exposure to the GFP-gC1qR vector decreased the proliferation of the cervical squamous carcinoma cells. There was an apparent decrease in DNA synthesis when C33a and SiHa cells were exposed to the GFP-gC1qR vector for $48 \mathrm{~h}$ after the initial manipulation. The effects on cell proliferation were also determined following exposure to SB202190 + GFP-gC1qR vector or to plain medium. There was no difference in proliferation between the SB202190 + GFP-gC1qR vector group and the plain medium group $(p>0.05)$. This suggested that gC1qR-induced apoptotic cervical cancer cell death was related to activation of the p38 MAPK signalling pathway.

\section{Discussion}

The $\mathrm{gC1qR}$ is a multi-compartmental and multi-functional cellular protein that is expressed on a wide range of tissues and cell types, including lymphocytes, endothelial cells, dendritic cells, and platelets [11], a recent study by Dembitzer et al. demonstrates strong gC1qR staining by immunohistochemistry in squamous cell carcinoma [12]. However, our data demonstrated that strong gC1qR expression in normal cervix tissue, however, the expression of $\mathrm{gC} 1 \mathrm{qR}$ was significantly decreased in human cervical squamous cell carcinoma tissues. This finding suggested that the gC1qR may play a negative role in the survival of human cervical cancer cells.

The list of biological responses mediated by the $\mathrm{gC} 1 \mathrm{qR}$ is extensive, and $\mathrm{gC} 1 \mathrm{qR}$ functions play major roles in inflammation, infection and immune regulation $[13,14]$. When constitutively expressed in a normal murine fibroblast cell line, gC1qR induces growth perturbation and morphological abnormalities and initiates apoptosis [15]. Our studies found that when the $\mathrm{gC} 1 \mathrm{qR}$ gene is overexpressed in cervical squamous carcinoma cells, increased rates of apoptosis were observed. Recent cohort studies have shown that the $\mathrm{gC} 1 \mathrm{qR}$ protein is primarily localized in the mitochondrial matrix [16]. Increasing evidence suggests that gC1qR-induced mitochondrial dysfunction is linked to apoptosis initiated by cytotoxic factor, such as ROS production. These findings have focused attention on the role of $\mathrm{gC} 1 \mathrm{qR}$ protein localized in intracellular compartment. Kim KB et al. also demonstrates that $\mathrm{gC} 1 \mathrm{qR}$ was mainly found in intracellular compartments, a small portion was detected in a patched area of the plasma membrane [17], so it is possible that the biological changes of cervical squamous cell carcinoma induced by gC1qR up-regulation include cellular surface membrane pool as well in present study. Studies have shown that in certain cancer cells, the expression of the gC1qR can be targeted with gC1qR-binding peptides, thereby making the $\mathrm{gC} 1 \mathrm{qR}$ gene a potential target for the diagnosis and therapy of cancer [18]. Further, our results revealed that gC1qR-overexpressing cervical cancer cells activate the p38 MAPK signalling pathway and undergo apoptosis, as detected by the formation of cervical cancer cell apoptotic bodies (data not shown).

Because activated MAPKs are common components of the apoptotic program [19], the activated MAPK family member phospho-p38 MAPK was examined in cells treated with the GFP-gC1qR vector, empty vector, or plain medium. Data shows that the phospho-p38 MAPK level was notably increased after GFP-gC1qR vector transfection for $48 \mathrm{~h}$. Pretreatment 
with the p38 MAPK inhibitor SB202190 reversed the decreases in viability, migration and proliferation of the gC1qR-overexpressing cervical squamous cancer cells. These results suggest that the activation of p38 MAPK was related to the gC1qR-induced apoptotic cell death of C33a and SiHa cells.

In conclusion, we demonstrate that the level of expression of the gC1qR gene plays an important role in cervical cancer cell apoptosis. Future studies are warranted to validate approaches for the promotion of the pathological processes mediated by the gC1qR. Therefore, the veracity of the in vitro studies described in the present data would have to be validated using suitable animal models in which the gene is overexpressed.

\section{Acknowledgments}

This study was supported by grants from the National Natural Science Foundation of China (No. 81000251), National Natural Science Foundation of China (No. 81272872), Nanjing Health Bureau Medical Science Foundation (No. YKK09090), Science and Technology Commission Foundation of Qingdao Open Economic Zone (No. 2009-2-28) and the Nanjing Medical Science and Technique Development Foundation.

\section{Conflict of interest}

The authors declare that they have no competing interests. The authors alone are responsible for the content and writing of the paper.

\section{References}

1 Parkin DM, Bray F, Ferlay J, Pisani P: Estimating the world cancer burden: Globocan 2000. Int J Cancer 2001;94:153-156.

2 Jemal A, Siegel R, Ward E, Murray T, Xu J, Smigal C, Thun MJ: Cancer statistics. CA Cancer J Clin 2006;56:106-130.

- G Gao LJ, Guo SY, Cai YQ Gu PQ, Su YJ, Gong H, Liu Y, Chen C: Cooperation of decay-accelerating factor and membrane cofactor protein in regulating survival of human cervical cancer cells. BMC Cancer 2009;9:384388.

4 Patel S, Chiplunkar S: Host immune responses to cervical cancer. Curr Opin Obstet Gynecol 2009;21:54-59.

5 Andersson S, Hellstr AC, Ren ZP, Wilander E: The carcinogenic role of oncogenic HPV and p53 gene mutation in cervical adenocarcinomas. Med Oncol 2006;23:113-119.

6 Kim JH, Kang JW, Kim MS, Bak Y, Park YS, Jung KY, Lim YH, Yoon DY: The apoptosis effects of the flavonoid N101-2 in human cervical cancer cells. Toxicol In Vitro 2012;26:67-73.

-7 Rubinstein DB, Stortchevoi A, Boosalis M, Ashfag R, Ghebrehiwet B, Peerschke EI, Calvo F, Guillaume $\mathrm{T}$ : Receptor for the globular heads of $\mathrm{C} 1 \mathrm{q}$ (gC1q-R, p33, Hyaluronan-binding protein) is preferentially expressed by adenocarcinoma cells. Int J Cancer 2004;110:741-750.

-8 Yao ZQ, Waggoner SN, Cruise MW, Hall C, Xie X, Oldach DW, Hahn YS: SOCS1 and SOCS3 are targeted by hepatitis C virus core/gC1qR ligation to inhibit T-cell function. J Virol 2005;79:15417-15429.

-9 Peerschke EI, Minta JO, Zhou SZ, Bini A, Gotlieb A, Colman RW, Ghebrehiwet B: Expression of gC1qR/p33 and its major ligands in human atherosclerotic lesions. Mol Immunol 2004;41:759-766.

$\checkmark 10$ Chowdhury AR, Ghosh I, Datta K: Excessive reactive oxygen species induces apoptosis in fibroblasts: role of mitochondrially accumulated hyaluronic acid binding protein 1 (HABP1/p32/gC1qR). Exp Cell Res 2008;314:651-667.

11 Meenakshi J, Goswami SK, Datta K: Constitutive expression of hyaluronan binding protein 1 (HABP1/p32/ $\mathrm{gC1qR}$ ) in normal fibroblast cells perturbs its growth characteristics and induces apoptosis. Biochem Biophys Res Commun 2003;300:686-693. 
12 Dembitzer FR, Kinoshita Y, Burstein D, Phelps RG, Beasley MB, Garcia R, Harpaz N, Jaffer S, Thung SN, Unger PD, Ghebrehiwet B, Peerschke EI: gC1qR Expression in Normal and Pathologic Human Tissues: Differential Expression in Tissues of Epithelial and Mesenchymal Origin. J Histochem Cytochem 2012;60:467-474.

$\$ 13$ Peerschke EI, Murphy TK, Ghebrehiwet B: Activation-dependent surface expression of gC1qR/p33 on human blood platelets. Thromb Haemost 2003;89:331-339.

14 Peerschke EI, Ghebrehiwet B: The contribution of gC1qR/p33 in infection and inflammation. Immunobiology 2007;212:333-342.

$15 \mathrm{Xu}$ L, Xiao N, Liu F, Ren H, Gu J: Inhibition of RIG-I and MDA5-dependent antiviral response by gC1qR at mitochondria. Proc Natl Acad Sci USA 2009;106:1530-1535.

16 Lührmann A, Nogueira CV, Carey KL, Roy CR: Inhibition of pathogen-induced apoptosis by a Coxiella burnetii type IV effector protein. Proc Natl Acad Sci USA 2010;107:18997-19001.

17 Kim KB, Yi JS, Nguyen N, Lee JH, Kwon YC, Ahn BY, Cho H, Kim YK, Yoo HJ, Lee JS, Ko YG: Cell-surface receptor for complement component $\mathrm{C} 1 \mathrm{q}(\mathrm{gC} 1 \mathrm{qR})$ is a key regulator for lamellipodia formation and cancer metastasis. J Biol Chem 2011;286:23093-23101.

-18 Mallick J, Datta K: HABP1/p32/gC1qR induces aberrant growth and morphology in Schizosaccharomyces pombe through its N-terminal alpha helix. Exp Cell Res 2005;309:250-263.

19 Wu RW, Yow CM, Wong CK, Lam YH: Photodynamic therapy (PDT)-Initiation of apoptosis via activation of stress-activated p38 MAPK and JNK signal pathway in H460 cell lines. Photodiagnosis Photodyn Ther 2011;8:254-263. 